\title{
Retail Location Choice with Complementary Goods: An Agent-Based Model
}

\author{
Arthur Huang and David Levinson \\ Department of Civil Engineering, University of Minnesota \\ 500 Pillsbury Drive SE., Minneapolis, MN 55455 \\ \{huang284, dlevinson\}@umn.edu
}

\begin{abstract}
This paper models the emergence of retail clusters on a supply chain network comprised of suppliers, retailers, and consumers. Firstly, an agent-based model is proposed to investigate retail location distribution in a market of two complementary goods. The methodology controls for supplier locales and unit sales prices of retailers and suppliers, and a consumer's willingness to patronize a retailer depends on the total travel distance of buying both goods. On a circle comprised of discrete locations, retailers play a non-cooperative game of location choice to maximize individual profits. Our findings suggest that the probability distribution of the number of clusters in equilibrium follows power law and that hierarchical distribution patterns are much more likely to occur than the spread-out ones. In addition, retailers of complementary goods tend to co-locate at supplier locales. Sensitivity tests on the number of retailers are also performed. Secondly, based on the County Business Patterns (CBP) data of Minneapolis-St. Paul from US Census 2000 database, we find that the number of clothing stores and the distribution of food stores at the zip code level follows power-law distribution.
\end{abstract}

Keywords: clustering, agent-based model, location choice, distribution pattern.

\section{Introduction}

In economic geography, clusters are geographical agglomerations of firms with similar or complementary capabilities [1]. Geographical clusters of business locations have been prominent phenomena in almost all countries and regions. Global integration increasingly contributes to regional specialization, with decreasing transportation costs and trade barriers enabling firms to closely interact with other firms to benefit from local economies of scale 23 .

An early investigation of clustering was performed by Marshall 4, who argued that while firms are directly connected through business exchange, they are also indirectly linked through competition for labor and production factors, and that clustering of locations represented the distribution of economic activities. Weber [5] proposed a theory of industrial location where industrial organizations locate to minimize transportation costs of raw materials and final product. Christaller,

J. Zhou (Ed.): Complex 2009, Part I, LNICST 4, pp. 175-187, 2009.

(C) ICST Institute for Computer Sciences, Social Informatics and Telecommunications Engineering 2009 
in his central place theory [6], indicated that the effects of market threshold and consumers' preferences in terms of range of patronizing lead to a system of central places, wherein each center supplies certain types of products forming levels of hierarchy. Krugman [7] asserted that the geographical distribution of firms is balanced by centripetal and centrifugal forces. The centripetal forces entice firms to cluster, and the centrifugal forces cause firms to scatter.

The mechanism of business clustering has gained increasing attention. Porter [8] formulated a diamond model to identify the mechanism of fostering industrial dynamism and long-term development. Levinson and Krizek 9] proposed four factors impacting a firm's decision of where to locate on a spatially-structured supply chain networks: complementors, competitors, connectors, and customers, which comprised the diamond of exchange. Huang and Levinson [10] studied retail location choice on a supply chain network of one product and found that different numbers of competitors and transportation can lead to different retail distribution patterns.

Hierarchical distributions of resources and economic activities have been widespread in almost every city, region, and nation. Power-law distributions have been found to well fit many natural and social phenomena, such as the population of cities [12, distribution of land uses [13, and the number of citations received by published academic papers [14. Zipf [15] proposed that city sizes follow a special form of the hierarchical distribution which is latter named as the Zipf's law. Gabaix [16 and Ioannide et al. 17] indicated that the rankdistribution of US cities follows the Zipf's law. Zipf's law was also found to fit the distribution of US firm sizes and the sales of US manufacturing firms [1819]. Similar results are also found for European and Japanese firms [20 21 22].

Yet human beings are still lacking understanding about the micro-foundations of the agglomeration of human activities and resources; quantitative theoretical models that can properly answer the question of how and why clusters emerge and prosper need to be formulated. Microscopically, clusters form by experiencing a complex and self-organized process in developmental stages, where business agents are constantly learning and adapting [23. It is of interest to examine how individual agents' seemingly random and chaotic decisions and interactions as a whole lead to clusters of firms.

As an extension of Huang and Levinson [10, this research builds an agentbased model to examine retail location choice on a simplified supply chain network of suppliers, retailers, and consumers. Retailers maximize profits by locating, which is modeled as a repetitive game. We are interested in the retail distribution pattern in equilibrium. It should be noted that this study analyzes a pure model of agglomeration of firms without forms of co-operation and other inter-organizational linkages. The basic assumptions of this model are: (1) Two categories of products exist in the market; one retailer only sells one category of products. (2) Each consumer needs both products. (3) Each consumer buys all needed products of one category from one retail=er in one trip. (4) Consumers share the same utility function, suggesting that they have the same taste when patronizing retailers. (5) Suppliers of the same product offer the same unit 
sales price and keep their price and locations fixed at all times. (6) Retailers of the same product have the same fixed unit sales prices. (7) Retailers' moving is costless.

The rest of the paper is organized as follows. Section 2 describes autonomous players and defines the concepts of cluster and average cluster density to measure retail spatial patterns. Section 3 depicts and analyzes the simulation results. Section 4 performs sensitivity tests on the number of retailers. Section 5 discusses the principles of retail location choice. Section 6 analyzes the distribution patterns of clothing stores and food stores in Minneapolis-St. Paul in the state of Minnesota, United States. Finally, Section 7 concludes the paper.

\section{The Model}

In this section, a multi-agent paradigm is adopted to model a repetitive noncooperative game of retail location choice. All players sit on the circle of a finite number of uniform locations. This section introduces agents in this model: suppliers, retailers, and consumers. Variables and constants used in this research are listed in Table 1 .

\subsection{Consumers}

The market has two categories of products $x$ and $y$, which are sold by two kinds of retailers. Let $R_{x i}$ indicate retailer $i$ of product $x$, and $R_{y j}$ indicate retailer $j$ of product $y$. Consumers hope to buy both products with minimum cost, which, in this research, implies minimum total travel distance. A trip is defined as a round-trip from home to visit $R_{x i}$ and $R_{y j}$. Trips are assumed not to have fixed costs; only total distance matters. In the scenario of $W_{x}$ number of $R_{x i}$ and $W_{y}$ number of $R_{y j}$, there are in total $W_{x} \cdot W_{y}$ trip candidates, from which a consumer chooses the shortest trip.

Let $d_{p i}, d_{p j}$, and $d_{i j}$ respectively denote the shortest distance between consumer $p$ and retailer $R_{x i}$, between consumer $p$ and $R_{y j}$, and between $R_{x i}$ and $R_{y j}$. Since players locate on a circle, it can be easily deduced that $d_{p i}, d_{p j}$, and $d_{i j}$ are larger than or equal to the half of the circle perimeter. Given retailer $R_{x i}$ and retailer $R_{y j}$, the shortest trip distance $d_{t}$ for consumer $p$ can be calculated by the following method: if the summation of $d_{p i}, d_{p j}$, and $d_{i j}$ is larger than or equal to the perimeter of the circle where retailers are located, the shortest trip is the perimeter of the circle. Otherwise, the shortest trip distance equals twice of the largest value of $d_{p i}, d_{p j}$, and $d_{i j}$.

Consumers are assumed to be homogeneous, sharing the same utility function. The utility for consumer $p$ to patronize retailer $R_{x i}$ equals:

$$
U_{p i}=\sum_{t=1}^{W_{x} \cdot W_{y}} k_{1} \cdot d_{t}^{\beta} \cdot \pi_{t i}
$$

Where $\beta$ is expected to be negative because longer travel distance generally diminishes consumers' willingness of patronizing. To account for preferences 
Table 1. List of Variables

\begin{tabular}{ll}
\hline \hline Variables & Description \\
\hline$d_{p i}$ & shortest distance between consumer $p$ and retailer $R_{x i}$ \\
$d_{p j}$ & shortest distance between consumer $p$ and retailer $R_{y j}$ \\
$U_{p i}$ & consumer $p$ 's utility of partronizing retailer $R_{x i}$ \\
$\pi_{t i}$ & dummy variable, equaling 1 if Retailer $R_{x i}$ is included in trip $t$ \\
$d_{t}$ & total travel distance of trip $t$ \\
$b_{p i}$ & binary variable, which equals 1 if consumer $p$ patronizes retailer $R_{x i}$ \\
$\rho_{p i}$ & probability for consumer $p$ to patronize retailer $R_{x i}$ \\
$\rho_{p m}$ & probability for consumer $p$ to patronize a retailer sitting at locale $m$ \\
$m_{i k}$ & shortest distance between retailer $R_{x i}$ and supplier $k$ of product $x$ \\
$\Omega_{i m}$ & expected profit for retailer $R_{x i}$ when locating at $m$ \\
$\Pi_{i}$ & actual profit of retailer $R_{x i}$ \\
$\sigma_{m k}$ & shortest distance between supplier $k$ and locale $m$ on the circle \\
$l_{m k}$ & binary variable, which equals 1 if a retailer in location $m$ patronizes supplier $k$ \\
$l_{i k}$ & binary variable, which equals 1 if retailer $R_{x i}$ patronizes supplier $k$ \\
$\epsilon_{i}$ & number of retailers in cluster $i$ \\
$\tau_{i}$ & number of locations in cluster $i$ \\
$\varphi_{n}$ & mean cluster density of the distribution pattern of $n$ retailers \\
\hline \hline Constants Description \\
\hline$k_{1}$ & a constant in consumers' uitlity function \\
$\theta$ & unit retail sales price of product $x$ \\
$\chi$ & unit retail $y$ sales price of product $y$ \\
$\lambda_{x}$ & individual customer's demand on product $x$ \\
$\lambda_{y}$ & individual customer's demand on product $y$ \\
$u$ & retailers' unit shipping cost per product \\
$\delta$ & unit sales price of suppliers of $x$ \\
$v$ & unit sales price of suppliers of $y$ \\
$N$ & number of consumers \\
$K$ & number of suppliers of product $x$ \\
$L$ & number of suppliers of product $y$ \\
$W_{x}$ & number of retailers of product $x$ \\
$W_{y}$ & number of retailers of product $y$ \\
$C$ & total number of locales on the circle \\
\hline
\end{tabular}

associated with factors other than traffic cost (and thus avoid a deterministic model, which is a special case where travel cost dominates), we use a logit model in which the probability for a consumer to patronize a retailer depends on travel cost but has a random component. The probability for consumer $p$ to patronize retailer $R_{x i}$ is formulated as:

$$
\rho_{p i}=\frac{e^{U_{p i}}}{\sum_{j \in W_{x}} e_{j}^{U_{p i}}}
$$

Similar formulas can be established for retailer $R_{y j}$. It should be noted that when a consumer's locale contains both retailers of $x$ and retailers of $y$, the total travel distance becomes zero. In reality, there is always some distance between a consumer's home and a retail store. Thereby in this case, we set this intrazonal distance to be 0.25 of the distance between two adjacent locations on the 
circle, which is a typical empirical value used in regional transportation planning models.

The Roulette Wheel Selection method is adopted for a consumer to select a retailer. This approach suggests that a retailer with a larger $\rho_{p i}$ for consumer $p$ has a greater chance to be selected by this consumer. A consumer's probabilities of patronizing all retailers comprise his wheel of selection, which is updated in every round; and a spin of the wheel selects a retailer. The sequence for consumers to patronize retailers is randomly decided for each round.

\subsection{Suppliers}

There are two kinds of suppliers which sell product $x$ and product $y$, who are evenly distributed on the circle and are co-located. The model assumes that all suppliers offer the same unit sales price and can always produce enough goods to meet market demand. Suppliers locations are fixed in all rounds.

\subsection{Retailers}

Retailers connect suppliers and consumers on supply chains. Retailers' initial locations are randomly assigned. In the beginning of each round, retailers evaluate expected profits of all locations. For example, retailer $R_{x i}$ 's expected profit in locale $m, \Omega_{m}$, is calculated as:

$$
\Omega_{m}=\left(\sum_{p=1}^{N} \lambda \cdot \rho_{p m}\right) \cdot\left[\theta-\sum_{k=1}^{K}\left(\delta+u \cdot \sigma_{m k}\right) l_{m k}\right]
$$

Where $\sum_{p=1}^{N} \lambda \cdot \rho_{i m}$ represents total expected sales of products in locale $m$. The following part in brackets refers to expected profit per product, equaling sales price minus cost. A retailer's cost includes the purchasing cost of products from a supplier and the shipping cost which is proportional to shipping distance and quantity of products. Here we assume a retailer patronizes the closest supplier. After evaluating profits of all localities, a retailer moves to the locale that has the highest expected profit (revenue - cost), given others are geographically fixed at that time. Each retailer can only move once per round; the sequence for retailers to move is randomly decided, no matter what products they sell.

After all retailers choose locations, consumers begin to patronize retailers; the method is introduced in Section 2.1. Retailers' actual profits are calculated at the end of one round. A typical formula of retailer $R_{x i}$ 's actual profit, $\Pi_{x i}$, is as follows:

$$
\Pi_{i}=\left(\sum_{p=1}^{N} \lambda \cdot b_{p i}\right) \cdot\left[\theta-\sum_{k=1}^{K}\left(\delta+u \cdot m_{i k}\right) l_{i k}\right]
$$

Compared with function (3), the main difference is the way total sales amount is calculated, which in this function equals $R_{x i}$ 's sales price times actual sales amount at the end of a round. The actual profit and expected profit are different for two reasons: First, actual profit is calculated when all retailers have had an 
opportunity to relocate, that is at the end of a round, whereas expected profit may be estimated before other retailers find new locations. Second, consumers patronize retailers stochastically.

\subsection{Measuring Spatial Distribution}

We measure the number of discrete retail clusters and average cluster density when a game reaches equilibrium. A cluster is defined as an agglomeration of retailers that are geographically adjacent or in the same locale of the circle. Cluster density is calculated as the number of retailers in a cluster divided by the number of locations in the cluster. The mean average cluster density of $n$ retailers, $\varphi_{n}$, is formulated as:

$$
\varphi_{n}=\frac{1}{M} \sum_{i=1}^{M} \frac{\epsilon_{i}}{\tau_{i}}
$$

where $\tau_{i}$ is the number of locations in cluster $i ; \epsilon_{i}$ is the number of retailers in cluster $i ; M$ is the total number of clusters.

\section{Experiments and Results}

In our first basic experiment, all agents sit on a circle of 100 uniform discrete locations, where 5000 consumers are evenly distributed on these locations. A consumer's demand on product $x$ is 20 and on product $y$ is 10 . First, we examine the scenario of 5 retailers of $x, 5$ retailers of product $y, 5$ suppliers of $x$, and 5 suppliers of $y$. Table 2 shows the values of the parameters used in this experiment. A game is believed to have achieved an equilibrium when all retailers stay at their current locales for three consecutive rounds. Since multiple equilibria may exist in this game, we test 400 different retail initial location patterns.

Typically a stable pattern emerges after the first round. Our results find five retail location distribution patterns, which can be grouped into four categories by the number of clusters (it should be noted that here we consider the geographical patterns, although each individual retailers' final location may vary in each game depending on their initial patterns and the sequence of moving). The

Table 2. Value of parameters in the scenario of 10 retailers and 10 suppliers

\begin{tabular}{|cc|cc|}
\hline \hline Parameters & value & Parameters & value \\
\hline$N$ & 5000 & $k_{1}$ & 1 \\
$C$ & 100 & $\chi$ & 2.5 \\
$u$ & $0.08(\$)$ & $\delta$ & $1.5(\$)$ \\
$v$ & $1.0(\$)$ & $K$ & 5 \\
$\theta$ & 3.5 & $L$ & 5 \\
$\lambda_{x}$ & 20 & $W_{x}$ & 5 \\
$\lambda_{y}$ & 10 & $W_{y}$ & 5 \\
\hline \hline
\end{tabular}


Table 3. Probability distribution of the number of clusters and cluster density for the case of 10 retailers and 10 suppliers

\begin{tabular}{|cc|cc|}
\hline \hline Number of clusters Probability & \multicolumn{2}{|c|}{ Cluster density } & Probability \\
\hline 1 & 0.861 & 10 & 0.741 \\
2 & 0.128 & 6 & 0.120 \\
3 & 0.011 & 5 & 0.117 \\
& & 3.3 & 0.011 \\
& 3 & 0.011 \\
\hline \hline
\end{tabular}

probabilities for different numbers of clusters and cluster densities are shown in Table 3. The most common pattern is only one cluster, where all retailers accumulate at a supplier locale. All the retail distribution patterns share two features: (1) retailers only stay at supplier locales; (2) the same number of retailers of $x$ and retailers of $y$ co-locate, indicating that they constitute pairs. It is interesting to notice that the evenly distributed pattern of retailers - every one retailer of $x$ and every one retailer of $y$ double at a supplier locale - does not appear in our experiments. To further explore its possibility, we purposely set the initial distribution pattern to be very similar to the evenly distributed one, the result of which is that the evenly distributed pattern emerges.

It can be summarized that the hierarchical pattern of one cluster is most common, while the spread-out patterns with a larger number of clusters are very rare, which is the feature of power-law distribution. According to the definition of powerlaw distribution, a quantity $x$ follows a power law if its distribution obeys

$$
p(x) \propto x^{-\alpha},
$$

where $\alpha$ is the scaling parameter. The logarithmic scale of $p(x)$ and $x$ have the form of a linear relationship with slope $-\alpha$. Figure 1 shows log-log plots of

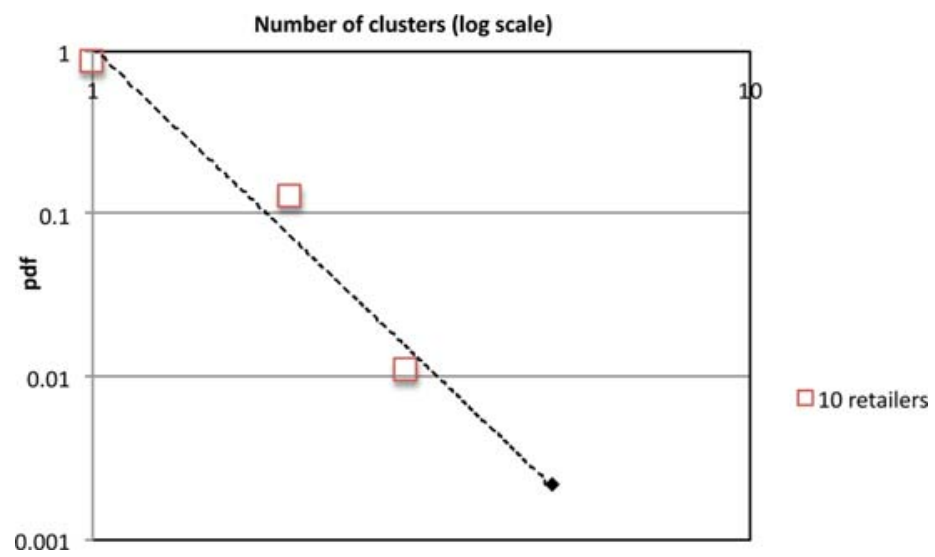

Fig. 1. Log-log plot showing power-law distributions in the number of clusters for 10 retailers ( 5 retailers of $x$ and 5 retailers of $y$ ), where $\alpha=3.83$ 


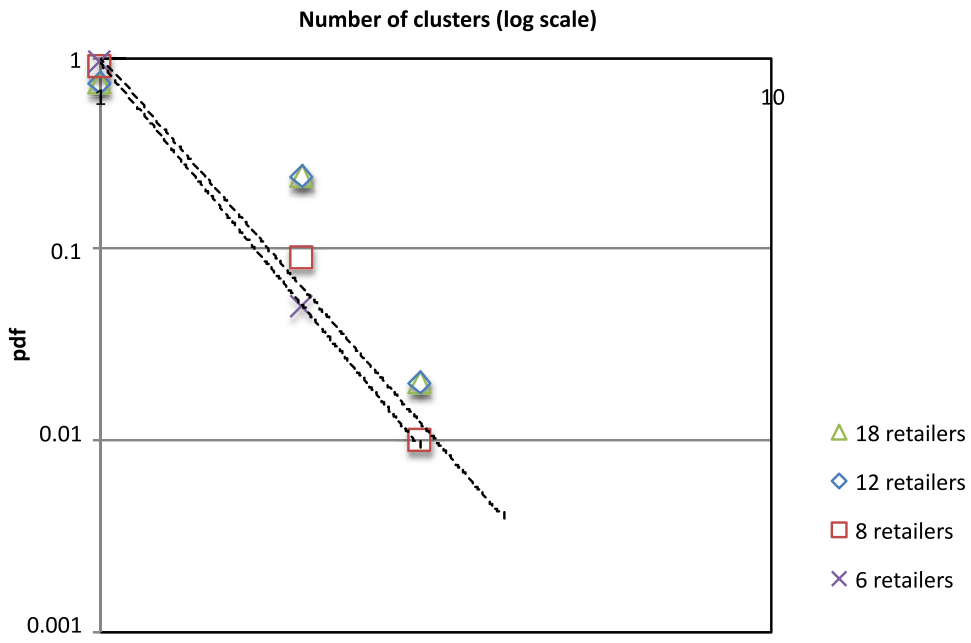

Fig. 2. Log-log plot showing power-law distributions in the number of clusters for the scenarios of $6,8,12$, and 18 retailers in total, where the number of retailers of $x$ equals the number of retailers of $y$

probability distribution of the number of clusters in our result. The model fit test confirms our hypothesis of power law; $\alpha$ is estimated to be 3.83 through maximum likelihood estimation (MLE).

\section{Sensitivity Tests on the Number of Retailers}

Our sensitivity tests investigate different numbers of retailers. First, presumably having the same number of retailers of $x$ and retailers of $y$, we examine the scenarios with total number of retailers respectively equaling $4,6,8,10,12$, $14,16,18$, and 20. 100 different retail initial location patterns are examined for each scenario; other conditions are the same as the first experiment. The experimental results disclose that the probabilities of the numbers of clusters in all these cases follow power-law distributions. The pattern of one cluster at a supplier locale has the highest probability to appear; in addition, each cluster contains the same number of retailers of $x$ and retailers of $y$. Figure 2 shows the probability distribution for the scenarios of $6,8,12,18$ retailers.

Second, we also examine the scenarios where the number of retailers of $x$ and retailers of $y$ are not equal. We set the number of retailers of $x$ to be 5 , and run the number of retailers of $y$ from 4 to 9; each case are tested with 100 different retail initial location patterns. The results, as shown in Figure 3, also indicate that hierarchical patterns emerge with a high probability. The probability distributions of the numbers of resultant clusters are all found to well fit the power-law distribution. Table 4 displays the estimated exponent $\alpha$ for the scenarios of various numbers of retailers. It is disclosed that controlling for the 


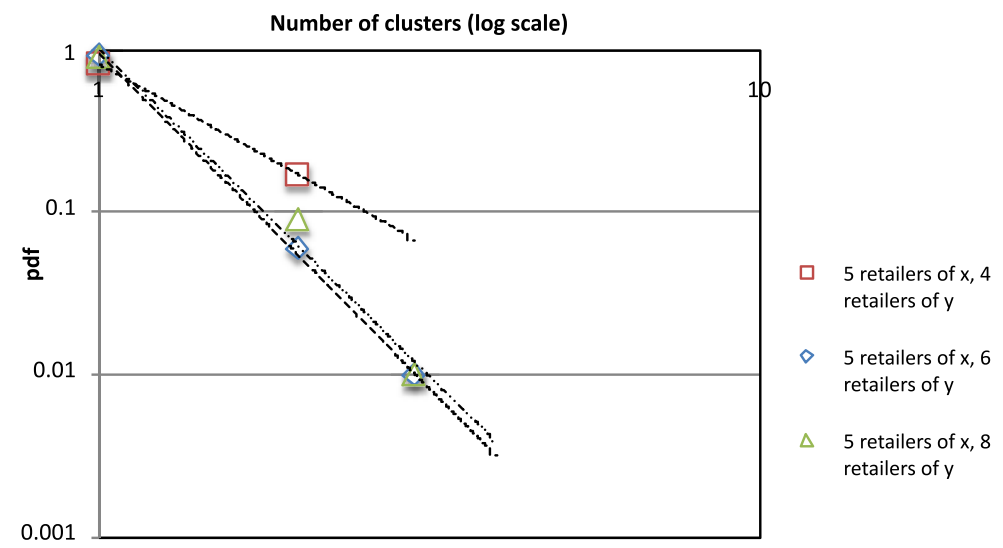

Fig. 3. Log-log plot showing power-law distributions in the number of clusters for the scenarios where the number of retailers of $x$ equals 5 and the number of retailers of $y$ respectively equals 4,6 , and 8 . When the number of retailers of $y$ equals $6, \alpha=2.27$; when the number of retailers of $y$ equals $6, \alpha=4.11$; when the number of retailers of $y$ equals $6, \alpha=4.03$.

Table 4. Estimated exponent $\alpha$ for different numbers of retailers

\begin{tabular}{|c|c|c|}
\hline \hline \# of retailers of $x$ & \# of retailers of $y$ & $\alpha$ \\
\hline 5 & 4 & 2.27 \\
5 & 5 & 3.81 \\
5 & 6 & 4.11 \\
5 & 7 & 4.58 \\
5 & 8 & 4.01 \\
5 & 9 & 4.01 \\
\hline 6 & 6 & 3.59 \\
7 & 7 & 3.77 \\
8 & 8 & 2.00 \\
9 & 9 & 3.10 \\
10 & 10 & 3.49 \\
\hline \hline
\end{tabular}

number of retailers of $x$, as the number of retailers of $y$ increases, the scaling parameter becomes larger, meaning that the slope of the power-law trend line gets steeper. Additionally, the values of the scaling parameter in the cases of different numbers of retailers of $x$ and $y$ tend to be larger than those of the scenarios with the same number of retailers of $x$ and $y$.

\section{Discussion}

Our experiments illustrate some principles for retail location choice in our model:

1. Pairing of retailers of complementary goods. Ceteris paribus, a retailer is more likely to move to a locale with more retailers of complementary goods. 
2. Staying close to suppliers. Retailers all co-locate with suppliers to reduce transportation cost.

3. Avoiding direct competition. All else equal, retailers choose places whose neighborhood has a lower density of retailers of the same product to avoid direct competition.

If there is only one product in the market, such hierarchical patterns cannot be stable in that some retailers in a big cluster can easily move to an open space on the circle to occupy a larger market [10]. In this model, however, since consumers consider total travel distance of buying both goods, retailers' location choice depends not only on their distance to suppliers and consumers, but also on the locations of retailers of complementary goods. Our results discover that a retailer does not unilaterally move to a new locale unless it can pair with another retailer of complementary goods there.

In the central place theory, Christaller 6] claimed that in the areas with evenly-distributed population and resources, settlements have equidistant spacing between centers of the same order; high-order services are farther away from low-order services. Yet this research reveals that even in a market of two equally important products, hierarchical distribution patterns can autonomously emerge. This comports with the notion of retail districts found in many cities 9], such as the Kappabashi district of Tokyo specializing in kitchen equipment (and plastic sushi) along with similar examples of clustered competitors. In this model, although the even distribution pattern of retailers can occur under certain circumstances, to acheive this each cluster needs almost the same timing to emerge, which has a high requirement for retail initial distribution conditions and the sequence of location choice. Thereby it is much more difficult to emerge than the hierarchical ones.

\section{Retail Geographical Distribution in the Twin Cities}

Based on the County Business Patterns (CBP) data from the US Census 2000 database, we further examine the retail geographical distribution patterns in the Twin Cities. Each zip code area is considered as a cluster. Two categories of retailers are selected according to the 6-digit NAICS code. One category is food and beverage stores, including supermarkets and other grocery (except convenience) stores (445110) and convenience stores (445120); we believe products in this category are complementary to each other. The other category is clothing and clothing accessories stores, which include: mens clothing stores (448110), womens clothing stores (448120), childrens and infants' clothing stores (448130), family clothing stores (448140), other clothing stores (448190), and shoe stores (448210); the commodities from these stores are also complementary.

Fig 4 presents the cumulative distribution for the number of food and beverage store establishments. The distribution fits the power law with an R-square of 0.94 , and the scaling parameter is estimated to be 1.10 . Fig. 5 shows the 


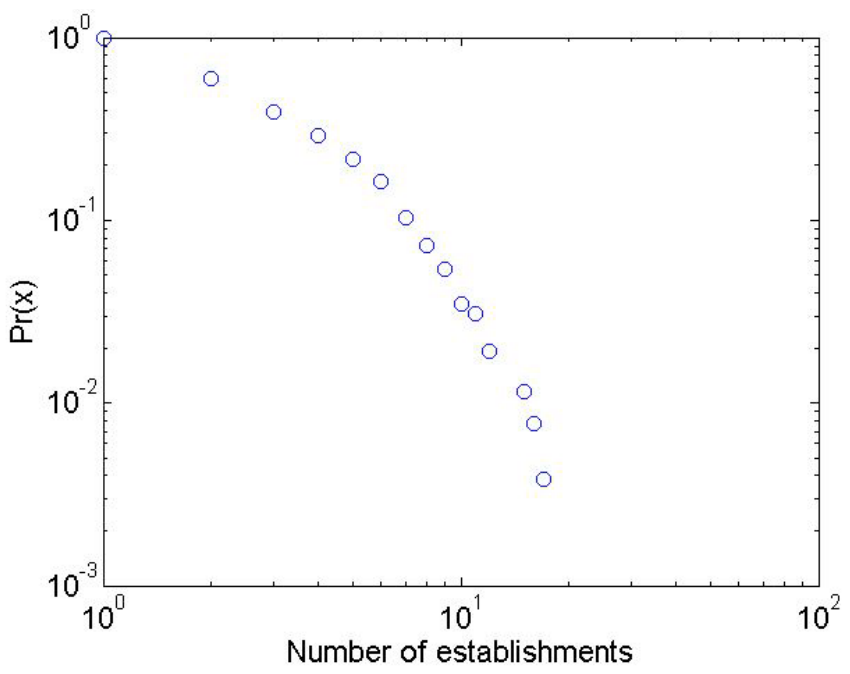

Fig. 4. The cumulative distribution for the number of food and beverage stores by zip code in the Twin Cities, MN in 2000. The scaling parameter is estimated to be 1.10.

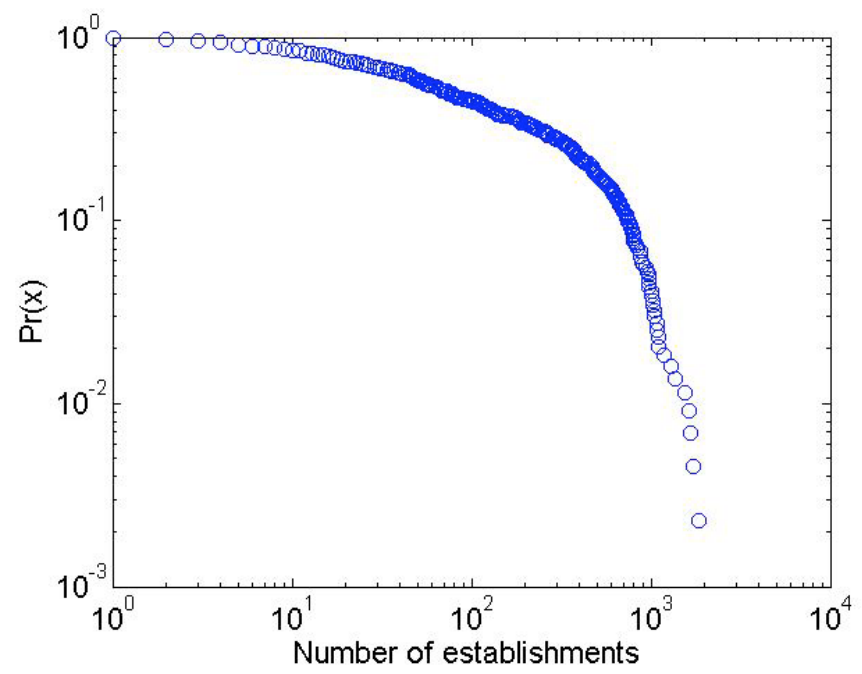

Fig. 5. The cumulative distribution for the number of clothing and accessories stores by zip code in the Twin Cities, MN in 2000. The scaling parameter is estimated to be 0.45 .

cumulative distribution for the number of clothing and clothing accessories stores. Its distribution is power law with a kink in the curve; the MLE method estimates the scaling parameter to be 0.45 . Such findings can be seen as empirical evidence of hierarchical distribution patterns of retailers of complementary goods. 


\section{Conclusions}

Geographical clusters have received great attention in recent years, yet the mechanism of clustering of business activities has not been fully understood.

This paper proposes an agent-based model to examine retail location choice in a market of complementary goods. This model considers the impact of both market demand and transportation cost. Our results find autonomous emergence of retail clusters; the hierarchical distribution patterns (in particular, the pattern of only one cluster) appear with a high probability. The probability distributions of the number of retail clusters follows power laws. It should be noted that this occurs with only a single mechanism (trip chaining for complementary goods on the part of the customer), and other mechanisms (such as the desire of customers to comparison shop) are not required to produce clusters, but may also be additional source of clustering behavior. In addition, based on the US Census data in 2000, we find that the retail distribution patterns of retailers of food stores and clothing stores by zip code follow power laws.

Retail location choice is a process involving balancing many different factors, such as distance to suppliers, distance to its complementary goods, and distance to direct competition. This research discloses that co-locating of retailers of complementary goods is a striking phenomenon. In addition, retailers settle down at their supplier locales to minimize transportation cost.

The framework of this model can be extended in the following aspects. Factors such as price, brand, product quality, word of mouth, and considerations about scheduling can impact consumers' choice of retailers. The model can incorporate mechanism by which retailers can compete by adjusting sales price and quality. Land price and local wage price also affect retailers' cost. Another intriguing future work is to examine this model in the context of a more general grid network, which requires modifying the rule of calculating travel distance and transportation cost. Also, it would be of interest to relax the assumption that one retailer can only sell one category of products; the model will better reflect the reality if retailers can autonomously decide whether to specialize or expand their scope.

In empirical studies, it is worthwhile to further consider what the proper level should be to examine the distribution of retailers. Most studies categorize industries and plants into politically defined regions such as states, counties, Metropolitan statistical areas (MSA), and zip code areas. While they can be meaningful indicators of clusters, clusters that are on the edges of several adjacent counties or zip code areas are not properly measured by looking from the county or zip code level.

\section{References}

1. Richardson, G.B.: The Organization of Industry. Economic Journal 82, 883-896 (1972)

2. Brakmann, S., Garretsen, H., van Marrewijk, C.: An Introduction to Geographical Economics. Cambridge University Press, Cambridge (2001) 
3. Baldwin, R., Forslid, R., Martin, P., Ottaviano, G., Robert-Nicoud, F.: Economic Geography and Public Policy. Princeton University Press, Princeton (2003)

4. Marshall, A.: Principles of Economics. Macmillan, London (1890)

5. Weber, A.: Theory of the Location of Industries. University of Chicago Press (1957)

6. Christaller, W.: Central Places in Southern Germany (Translated by C.W. Baskin). Prentice-Hall, NY (1966)

7. Krugman, P.: Urban concentration: The Role of Increasing Returns and Transport Costs. International Regional Science Review 19, 5-30 (1996)

8. Porter, M.: The Role of Location in Competition. International Journal of the Economics of Business 1, 35-40 (1994)

9. Levinson, D., Krizek, K.: Planning for Place and Plexus: Metropolitan Land Use and Transport. Routledge, New York (2008)

10. Huang, A., Levinson, D.: An Agent-based Retail Location Model on a Supply Chain Network. University of Minnesota. Working paper (2008)

11. Simon, H.A., Bonini, C.P.: The Size distribution of business firms. American Economic Review 48(4), 607-617 (1958)

12. Clauset, A., Shalizi, C., Newman, M.E.J.: Power-law Distributions in Empirical Data. SFI Working Paper (2007)

13. Andersson, C., Hellervik, A., Lindgren, K.: A Spatial Network Explanation for a Hierarchy of Urban Power Laws. Physica A 345, 227-244 (2005)

14. Redner, S.: How Popular is Your Paper? An Empirical Study of the Citation Distribution. European Physical Journal B 4, 131-134 (1998)

15. Zipf, G.: Human Behavior and the Principle of Least Effort: An Introduction to Human Ecology. Addison-Wesley, Reading (1949)

16. Gabaix, X.: Zipf's Law For Cities: An Explanation. Quarterly Journal of Economics 114, 739-767 (1999)

17. Ioannides, Y., Overman, H.: Zipf's Law for Cities: An Empirical Examination. Regional Science and Urban Economics 33, 127-137 (2003)

18. Axtell, R.: Zipf Distribution of U.S. Firm Sizes. Science 293, 1818-1820 (2001)

19. Stanley, M.: Zipf Plots and the Size Distribution of Firms. Economics Letters 49, 453-457 (1995)

20. Fujiwara, Y., Di Guilmi, C., Aoyama, H., Gallegati, M., Souma, W.: Do Pareto-Zipf and Gibrat laws Hold True? An analysis with European rms. Physica A: Statistical Mechanics and its Applications 335, 197-216 (2004)

21. Okuyama, K., Takayasu, M., Takayasu, H.: Zipf's Law in Income Distribution of Companies. Physica A 269, 125-131 (1999)

22. Mori, T., Nishikimi, K., Smith, T.: The Number-Average Size Rule: A New Empirical Relationship Between Industrial Location and City Size. Journal of Regional Science 48, 165-211 (2008)

23. Maskell, P., Malmberg, A.: Localised Learning and Industrial Competitiveness. Cambridge Journal of Economics 23, 167-185 (1999) 